\title{
Magnitude and Factors of Occupational Injury among Workers in Large Scale Metal Manufacturing Industries in Ethiopia
}

\author{
Yitagesu Habtu' ${ }^{1}$, Abera Kumie ${ }^{2}$, Worku Tefera ${ }^{2}$ \\ ${ }^{1}$ Department of Health Information Technology, Hossana College of Health Sciences, Hossana, Ethiopia \\ ${ }^{2}$ Faculty of Medicine, College of Health Sciences, Addis Ababa University, Addis Ababa, Ethiopia \\ Email: yitagesuh@yahoo.com, aberakumie2@yahoo.com,workutefera2000@yahoo.com
}

Received 8 September 2014; revised 18 October 2014; accepted 19 November 2014

Copyright @ 2014 by authors and OALib.

This work is licensed under the Creative Commons Attribution International License (CC BY). http://creativecommons.org/licenses/by/4.0/

c) $\underset{\mathrm{EY}}{0}$ Open Access

\section{Abstract}

Background: The burden of occupational injury in most developing countries including Ethiopia is becoming a public health problem. Therefore, information that shows the magnitude and predictors of occupational injury in most risky work places in Ethiopia such as large scale metal manufacturing industries is indispensable for proper health intervention programs. Objectives: The aim of this study was to assess the magnitude and factors affecting occupational injuries among workers engaged in large scale metal manufacturing industries in Addis Ababa, Ethiopia. Methods: Facility based cross sectional study was conducted among 829 workers engaged in large scale metal manufacturing industries in Addis Ababa, Ethiopia from February 1 to March 30, 2010. Fifty percent $(50 \%)$ of large scale metal manufacturing industries were selected by simple random sampling after stratification. Then, calculated sample size was allocated for each industry by probability to proportional sample size. Subjects were stratified by working sections and those who were directly engaged in the work were selected from each stratum by simple random sampling after preparing a frame from payroll of those industries. The data were collected by pretested structured questionnaire. Observational checklist and in-depth interview with key informants were held to triangulate the information with quantitative findings. Both bivariate and multivariate logistic regressions were done to identify factors of occupational injury. Results: The magnitude of occupational injury was 489 per 1000 exposed workers per year. Twenty nine percent of injured workers were hospitalized of which $98(82.4 \%)$ for 24 or more working hours. Sex of workers [AOR: 3.32, 95\% CI: $(1.88,5.85)$ ], safety and health supervision [AOR: $1.60,95 \% \mathrm{CI}:(1.03,2.60)]$, hours worked per week [AOR: $2.37,(95 \% \mathrm{CI}:(1.55,3.61)]$, cigarette smoking [AOR: 3.36, 95\% CI: $(1.73,6.50)]$ and presence of functional danger signs/posts [AOR: 2.65 , (95\% CI: $(1.67,4.19)]$ were significantly associated factors with magnitude of occupational injury. Conclusion: The burden of occupational injury in metal manufacturing industry is really significant public health problem. Emphasis should be given to provide health and safety services on sex of workers, safety and health supervision, hours worked per week, cigarette smoking and functional danger signs. 


\title{
Keywords
}

Occupational Injury, Large Scale Metal Manufacturing Industries, Ethiopia

\author{
Subject Areas: Epidemiology, Global Health, Public Health
}

\section{Introduction}

Occupational injury is a global public health burden in terms of disability adjusted life year which contributed to $1.5 \%$ of all causes with estimated economic loss of $5 \%-10 \%$ growth national product [1] [2]. International labor organization conservatively estimated that about 2.3 million workers die each year from unintentional work related accidents and diseases [2] [3]. Morbidity also estimated to be more than 270 million people at work [2] [4]. People belonging to all economic groups suffer fatal injuries, but death rates due to injury tend to be higher in those developing countries where there is unsafe working environment [2] [4] less awareness [4] and trained workers, limited/no occupational services [5] psychosocial stress [6] [7] are prevailed.

In Ethiopia, there is scarcity of comprehensive data and nationwide researches on rate of occupational injuries and its factors in large scale metal manufacturing industries [8] [9]. The recent studies in small and medium scale industries showed that the occupational injury rate was 335/1000 workers exposed per year. Of these, the $17.1 \%$ of them being hospitalized with $40 \%$ of them for greater than 24 hours, 53.9\% absent from work, 191 days were lost due to injuries [10]. Another study in Afar showed that the overall prevalence rate was 783 per 1000 workers with the severity $11 \%$ is hospitalized and 6153 days lost due to injuries [11].

The knowledge on injured parts of the body with corresponding types of injury can help policy makers, managers, industrial hygienists, public health experts, initiators and job analysts to provide and design appropriate personal protective equipment and safe ergonomic design [12]-[16]. The commonest parts of the body injured in a study conducted in Akaki textile factory were fingers (42\%), lower leg (18.95\%), hands (13.3\%) [10]. Another study in Afar Regional State revealed that finger (32\%), lower leg (20.4\%), and eye (12.9\%) were among body parts commonly affected in that agricultural industry [11]. A study conducted on small and medium scale industries in Gondar Woreda similarly showed that hands (30\%), fingers (24\%), and eye (19\%) were commonly affected organs [10]. Reports compiled by MOLSA from 66 industries also showed that head (7\%), upper limb (47\%), lower limb (25\%) and trunk (8\%) were affected. Among industries, manufacturing industries including metal manufacturing industries hold first (91\%) of reported injuries with regard to parts of the body affected [8].

With regard to sources of injury, many studies showed that machinery, hand tools, and hit by falling objects, are the frequent causes [1] [15] [17] [18]. Study done at Gondar, in small and medium sized industries, showed that the commonest causes were machinery (23.9\%), splinting (21.7\%), hand tools (16.6\%) [10]. Another research in Tendaho agricultural industry indicated that, the commonest causes were hand tools (53.6\%), splinting/splashing objects (11.2\%), falling accidents (8\%), hit by falling objects (5.2\%) [11]. Reports from the ministry of labor and social affairs showed that the causes were machinery (31\%), style of loading/caring (18\%) and hand tools (11\%) [8].

Researchers showed that several factors were related to the occurrence, severity, and types of injury. Sociodemographic factors, working environment variables, workers' behavior and ergonomic related variables are risk factors for workers to be injured in workplace of manufacturing industries including metal manufacturing industries [10] [11] [13] [18]-[23].

Authors showed that age is significantly associated with occurrence of occupational injuries in which all showed the younger the age group the greater injury rate [10] [11] [19] [20]. Drinking alcohol [19] [22] [24], educational level [22]-[24], sleeping disorder [10] [11], job satisfaction [18] [22] [23], use of personal protective devices [11], hours worked per week [10] [11], health and safety training [10] [11], work experience [11] [22] [24], employment pattern [19] [22] [23], regular supervision [20] [22], smoking [19] [24], waste and protective maintenance [23] were statistically associated factors with magnitude of occupational injury. This study focused on determining magnitude and factors affecting occupational injuries in large scale metal manufacturing industries in Addis Ababa. The study will provide baseline information for policy makers to design strategy for pre- 
vention and control of occupational injuries to have healthy workforce and sound economic development. The information is also important for practitioners and researchers while it is in need.

\section{Methods}

\subsection{Study Setting and Design}

Institution based cross sectional study was conducted to assess the magnitude and factors of occupational injuries in workers engaged in large metal manufacturing industries in Addis Ababa from February 1 to March 30, 2010. In the city, of 634 large and medium scale manufacturing industries, 95 are metal processing and metal manufacturing industries. Out of these 51 are registered by ministry of trade and industry as large scale industry in the city [25].

\subsection{Study Size and Sampling Methods}

The sample size was calculated by using single population proportion formula with $50 \%$ expected prevalence, $5 \%$ the margin of error (d), $95 \%$ degree of confidence level $(\mathrm{Z} \alpha 2=1.96)$, design effect of $2 \%$ and $10 \%$ nonresponse rate to maximize the sample size. Those workers who had the chance of being selected were included in the study excluding administrative workers assuming that they are not exposed to factors of occupational injuries were study subjects. Number of manpower in 51 registered metal manufacturing industries in the city were counted to include those metal manufacturing industries which fulfill the criteria of large scale based on the operational definition of this study. The existence and functionality of these industries was checked from the records of Ministry of Industry and Trade. Then, those large scale metal manufacturing industries were stratified based on their expected risk similarity. Fifty percent of large scale metal manufacturing industries were selected by simple random sampling from those strata that fulfilled the criteria. The calculated sample size $(n=846)$ was allocated by probability proportional to size of each selected large scale metal manufacturing industries. The number allocated by PPS of each industry again was allocated by PPS of each section of selected industry which was stratified by working sections. Finally, study subjects were selected by simple random sampling method from payroll or lists from each working section of each stratified large scale metal manufacturing industries.

\subsection{Data Collection and Measurement}

Pretested structured questionnaire was used. Observational checklist was designed for evaluation of working environment, record review and employee's behavior on how to utilize personal protective devices during data collection. In depth interviews with key informants were held on thematic areas of occupational injury to triangulate the information with quantitative findings. One Bachelor Science in Environmental health, three Masters of public health students, one Bachelor Science in Engineering, two diploma in social science and two industrial safety inspectors were trained to collect data. Data collectors agreed to report and discuss their daily data collection activities to take immediate corrections and checking completeness until end of data collection and rearrange the code to prepare for data entry. Principal investigator and one supervisor supervised the data collection process and check completeness and consistency.

\subsection{Data Processing and Analysis}

Data were entered by using EPI INFO 3.5.1 version computer software package after editing and cleaning to check completeness and consistency. Finally, data were exported to SPSS for windows version 17 for data management and analysis. Descriptive statistics, binary logistic and multivariate logistic regression analysis were applied to identify the effect of factors on occupational injuries. Variables whose P values less than 0.30 at bivariate analysis were entered to multivariate analysis by using enter or standard method to avoid unstable estimates due to excess number of variables [26] [27].

\subsection{Operational Definition}

Occupational injury: Tissue damage from transfer to individuals of one of the five forms of energy or from accidents arising out of or in the course of employment but not includes work related diseases that need exposure assessment or laboratory tests and doctoral examination [28]. 
Manufacturing industry: An industry that processes physical or chemical materials or components into new products where the work is performed by power driven machine or hands [28] [29].

Large scale industry: Industry that employ 250 or more workers and uses power driven machines [8].

Severity of injury: Characterized by death, hospitalization more than 24 hours, and absence from work over 3 days in the last one year [10].

\subsection{Ethical Consideration}

The ethical approval and clearance was obtained from Addis Ababa University, College of public health and medicine institutional review board. Permission letter for all managers of selected industry was distributed. Data collectors requested for respondents' consent by the consent form consisting of confidentiality and autonomy of workers for the information they provide and range of participation. Health information was given after completion of the data collection and finding of the study was disseminated to those industries having problems.

\section{Result}

\subsection{Socio-Demographic Characteristics}

Out of 846 workers, 829 were participated in the study which gave the response rate of $97.99 \%$. Majority of participants, 720 (86.9\%) were males and 109 (13.1\%) were females. The median age of respondents was 32 with the minimum and maximum age of 18 and 61, respectively. Only 15 (1.8\%) of the study population were illiterate and the majority of the study population (73.0\%) have educational level of grade 9 and above. Majority of participants, 609 (73.5\%) were permanently employed while the rest 220 (26.5\%) were temporary workers.

\subsection{Distribution of Occupational Injury}

Four hundred five (48.9\%) of study participants had responded that they had incident at job that resulted occupational injury in the past 12 months giving an overall prevalence rate of 489 per 1000 exposed workers per year. Out of injured respondents, 119 (29.4\%) of participants had reported that they were hospitalized or stayed at home. A total of 3734 working days were lost due to injury.

Injured respondents were asked about body part affected, types, sources, days of the week of occurrence, and time of occurrence of injury. Study participants responded that hands 188 (46.4\%), finger 135 (33.3\%), toe 131 (32.3\%), and eye 112 (27.7\%) were commonly affected parts of the body. Abrasion or laceration 214 (52.8\%), eye injury 112 (27.7\%), cut 87 (21.5\%), puncture 76 (18.8\%) and dislocation 60 (14.8\%) were predominantly occurred types of injury.

Among injured workers who were assigned at different working sections, 65 (16.05\%), 52 (12.84\%) and 48 (11.85\%) were injured at machine area or crane operation, welding section, metal processing section, respectively.

\subsection{Distribution of Work Environment, Ergonomic and Behavioural Characteristics}

Regarding work environment factors, 561 (67.7\%) of respondents were at work for 48 or more hours while 268 (32.3\%) were less than 48 hours per week. Four hundred (57.1\%) of respondents had been regularly supervised at work about safety. Five hundred fifty five (66.9\%) had not ever taken safety and health training. Two hundred (34.0\%) of participants had work shift. Regarding ergonomic related factors, 384 (46.3\%), 333 (40.2\%), 229 (27.6\%), 381 (46.0), and 415 (50.1\%) of respondents revealed that availability of devices to move or lift objects, safely designed storage of materials, functionally danger signs, safely guarded machines and timely maintained machines, respectively.

Concerning behavioral factors, 288 (34.7\%), 142 (17.1\%), and 119 (14.4\%) of participants were used to drink alcohol, chew khat, and smoke cigarette, respectively. The study also showed that 89 (10.7\%) respondents had sleeping disorder at work places. Two hundred (24.2\%) participants were not satisfied with the current job and 113 (13.6\%) respondents had medical problems like extremity problem 48 (42.5\%), partial deafness 14 (12.4\%), and 9 (7.9\%) were specified medical problems and 47 (41.6\%) were commonly mentioned medical problems. Regarding availability of personal protective equipment, 483 (58.3\%) participants used personal protective devices at work places. 


\subsection{Observation of Work Environment}

Six occupational hazards were evaluated based on the operational definition given on the observational checklist in 28 working sections by injury matrix table of eight selected industries. Based on that, 23, 27 and 19 were with excessive heat, excessive noise and excessive dust, respectively. As result, only four of 28 sections had warning signs and posts of which three showed 25 lack protective devices. Only three were using devices and four of them had arranged materials in a protective manner from injury. Among eight industries, only one had completed the required occupational health services such as specified preventive measures, copy of safety and health regulation, safety and health personnel and written plan of safety and health services. Three out of eight industries had complete first aid equipment.

\subsection{Results of Qualitative Method}

A total of eight key informants were participated in the study on six essential issues to triangulate with the quantitative findings. A minimum age of 42 and maximum age of 58 are participated. So they were asked to respond on the following themes.

Almost all participants stated that the occupational injury is a major and sever health problem in the working areas that varies in different working sections of each industry as sources, factors and reasons of occupational injuries. As mentioned by participants, the most common sources of injury were machines and splintering objects. From experiences most of them reported that they usually had got injury by not using personal protective devices, unsafe work environment, irresponsible company owners for workers' health, old or unguarded and not timely maintained machines, overburdened working environment, unsafe solid waste management, shortage of health and safety education, workers' unsafe act, and having less experiences.

Regarding factors, most of participants quoted that most factors mentioned such as sex of workers, safety and health supervision, hours worked per week, cigarette smoking, presence of functional danger signs may be associated factors with magnitude of occupational injury from experiences. Except one participant, most participants reported that personal protective devices were limited or not available at all for some workers and may not be utilized by the users either they are not fit for each workers or unaware behavior of the workers.

\subsection{Factors Associated Occupational Injury}

Among selected variables, those that showed a significant association with occupational injury when adjusted for all variables are presented here. Males were 3.32 times more likely to be injured when compared to females [AOR: 3.32, 95\% CI: $(1.88,5.85)$ ].

Smokers were 3.36 times more likely to be injured when compared to non smokers [AOR: 3.36, 95\% CI: $(1.73,6.50)]$. Participants who were engaged to work 48 hours or more per week were 2.37 times more likely to be injured compared to those who were engaged to work for less than 48 hours per week [AOR: 2.37, 95\% CI: $(1.55,3.61)]$. Workers without health and safety supervision were 1.60 times more likely to be injured than those who were supervised [AOR: 1.60, 95\% CI: $(1.03,2.60)]$. Participants who were assigned at a work environment lacking functional danger signs were 2.65 times more likely to be injured than opposite work environment [AOR: 2.65, 95\% CI: $(1.67,4.19)$ ] (Table 1).

\section{Discussion}

Occupational injury is a global public health burden and economic burden in addition to other public health challenges in both industrialized and less industrialized country [1] [2].

The overall prevalence of occupational injury in this study was 489 per 1000 exposed workers per year. Studies in Asian pacific countries, Vietnam, Japan and New Zealand showed the magnitude of occupational injuries was 583, 385, 132 workers per 1000 per year in Small and medium sized manufacturing industries [1] [19] [30]. A study conducted in Small and medium sized manufacturing industries in Gondar showed the prevalence was 335 workers per 1000 exposed workers per year [10]. Findings by observational and key informants' interview supported the quantitative result of this study.

Regarding to severity which was measured by hospitalization/staying on bed and days away from work, this study showed the most severe condition than other findings [10] [11] [31] with 119 (29.4\%) hospitalization or stayed at home bed with 98 (82.4\%) for 24 or more working hours and 3734 working days were lost due to 
Table 1. Final model adjusted for variables on occupational injury among workers in large scale metal manufacturing industry in Addis Ababa, March, 2010.

\begin{tabular}{|c|c|c|c|c|}
\hline \multirow{2}{*}{ Variables } & \multicolumn{2}{|c|}{ Occupational injury } & \multirow{2}{*}{ COR (95\% CI) } & \multirow{2}{*}{ AOR $(95 \% \mathrm{CI})$} \\
\hline & Yes & No & & \\
\hline \multicolumn{5}{|l|}{ Sex } \\
\hline Male & 382 & 338 & $4.23(2.61,6.85)$ & $3.32(1.88,5.85)$ \\
\hline Female & 23 & 86 & 1 & 1 \\
\hline \multicolumn{5}{|l|}{ Educational status } \\
\hline Illiterate & 9 & 6 & $2.33(0.81,6.67)$ & \\
\hline Read and write & 26 & 15 & $2.69(1.38,5.24)$ & \\
\hline Primary school (1 - 8) & 101 & 67 & $2.34(1.62,3.39)$ & \\
\hline Secondary school (9 - 12) & 115 & 97 & $1.84(1.31,2.58)$ & \\
\hline Diploma or higher & 154 & 239 & 1 & \\
\hline \multicolumn{5}{|l|}{ Safety supervision } \\
\hline Yes & 159 & 314 & 1 & 1 \\
\hline No & 246 & 110 & $4.42(3.29,5.93)$ & $1.60(1.03,2.60)$ \\
\hline \multicolumn{5}{|l|}{ Hours worked per week } \\
\hline$<48$ hours & 68 & 200 & 1 & 1 \\
\hline$>48$ hours & 337 & 224 & $4.43(3.20,6.11)$ & $2.37(1.55,3.61)$ \\
\hline \multicolumn{5}{|l|}{ Functional danger signs } \\
\hline Yes & 42 & 187 & 1 & 1 \\
\hline No & 363 & 237 & $6.82(4.69,9.89)$ & $2.65(1.67,4.19)$ \\
\hline \multicolumn{5}{|l|}{ Safety training } \\
\hline Yes & 96 & 178 & 1 & \\
\hline No & 309 & 246 & $2.33(1.73,3.14)$ & \\
\hline \multicolumn{5}{|l|}{ Work shift } \\
\hline Yes & 137 & 145 & 1 & \\
\hline No & 268 & 279 & $1.02(0.76,1.36)$ & \\
\hline \multicolumn{5}{|l|}{ Cigarette smoking } \\
\hline Yes & 96 & 23 & $5.42(3.36,8.74)$ & $3.36(1.73,6.50)$ \\
\hline No & 309 & 401 & 1 & 1 \\
\hline \multicolumn{5}{|l|}{ Chewing chat } \\
\hline Yes & 96 & 46 & $2.55(1.74,3.74)$ & \\
\hline No & 309 & 378 & 1 & \\
\hline \multicolumn{5}{|l|}{ Drinking alcohol } \\
\hline Yes & 159 & 129 & $1.48(1.11,1.97)$ & \\
\hline No & 246 & 295 & 1 & \\
\hline \multicolumn{5}{|l|}{ Sleeping disorder } \\
\hline Yes & 62 & 27 & $2.66(1.65,4.27)$ & \\
\hline No & 343 & 397 & 1 & \\
\hline \multicolumn{5}{|l|}{ Job satisfaction } \\
\hline Yes & 282 & 346 & $0.52(0.37,0.72)$ & \\
\hline No & 123 & 78 & 1 & \\
\hline \multicolumn{5}{|l|}{ Use of personal protective devices } \\
\hline Yes & 198 & 285 & $0.47(0.35,0.62)$ & \\
\hline No & 207 & 139 & 1 & \\
\hline
\end{tabular}


injury. This also correlated from findings of key informants interviews.

This study showed that abrasion or laceration 214 (52.8\%), eye injury 112 (27.7\%), cut 87 (21.5\%), puncture 76 (18.8\%) and dislocation 60 (14.8\%) were five commonest types of injury. Studies in East Asia showed consistent result [1] [19] [30]. Other studies in Ethiopia, Gondar on small and medium scale industry, and Addis Ababa in textile factory workers and Afar showed consistent result [10] [11] [32] except that eye injury was higher in this result. This could be mainly by foreign metal chips which can splinter towards eye and poor utilization of goggles (13.2\%) in these industries.

Five commonest parts of the body injured in this study. Hands 188 (46.4\%), finger 135 (33.3\%), toe 131 (32.3\%), eye 112 (27.7\%) and back 52 (12.8\%). This finding is consistent with other findings in Ethiopia [16] [31] [32] and other studies in East Asia [1] [19] [30] in terms of their types although figures vary. This study showed machinery 169 (41.7\%), splintering objects 155 (38.3\%), Hit by falling objects 58 (14.3\%), hand tools 43 (10.6\%), and hot substances 39 (9.6\%) were among commonest sources of injury. Similarly, this study is in agreement with other studies which pointed out machinery, hand tools, and hit by falling objects, are the frequent causes in most industrial setups [1] [10] [15] [17] [18]. The causes are also specified in key informants result consistently with this quantitative finding.

Most Researchers showed that several factors were related to the occurrence, severity, and types of injury. Socio-demographic factors, working environment variables, worker's behaviour and ergonomic factors are the possible risk factors for workers to be injured in workplace of manufacturing industries including metal manufacturing industries [10] [11] [13] [17] [18] [20] [22] [33].

Sex of the worker showed significant association with magnitude of occupational injury when it is adjusted for all variables. This finding is consistent with other studies [10] [19] [22] [23] [32]. Some findings showed that age is significantly associated with magnitude of occupational injuries in which all showed the younger the age group the greater injury rate [10] [11] [19] [30]. However, this study showed that age is not significantly associated with occupational injury. This could be explained by young workers might be assigned at less machine areas as it need experiences in this study. Educational status is also was not significantly associated with magnitude of occupational injuries when adjusted all variables of interest. This is not also consistent with the study [22] [23]. This implied that education may not a guarantee for not being injured but safe practice.

Regarding the work environment factors, health and safety supervision was significantly associated with occupational injury when it is adjusted for all variables. This correlates with other studies [19] [22]. Similarly, hours worked per week were also showed significant association with occupational injury. This result is also in agreement with study done in Ethiopia [10] [11]. Safety and health training did not show significant difference when it is adjusted for all variables. However, this study is not consistent with other findings [10] [11]. This could be those workers who were not injured might respond as if they did not take due to the logic that they perceived they will take if they say no.

All behavioural and ergonomic related factors were significantly associated with magnitude of occupational injury in bivariate analysis but only cigarette smoking and presence of functional danger signs were associated after adjustment for all variables. This study for cigarette smoking is consistent with study done in Japan [13] but not for drinking alcohol [11] [19], sleeping disorder [10] [11], job satisfaction [10] [11] [18] [23], and use of PPE [11]. This might be due to workers may not want to express this personal behaviours at a time of data collection. Other literatures [4] [13] [15] [22], stated ergonomic related factors are predictors for occupational injuries although as to my knowledge there is no study showing these relation in Ethiopia until this study is completed.

\section{Limitation of the Study}

Since this study was a cross sectional study design study participants may not recalled occurrence(s) of occupational injury during one year period so that this may underestimate the overall prevalence. Annual leave, injured workers at home and low production seasons in industries may be responsible for underestimation of overall prevalence and unable to detect associations. Study participants might also perceive that responding as injured might bring benefits and failure to report socially sensitive information.

\section{Conclusion}

The overall lesson that we found from the research is that prevalence of occupational injury and severity is sig- 
nificantly high among workers engaged in large scale metal manufacturing industries in Addis Ababa. This magnitude implied that occupational health service coverage in these workplaces needs a due attention for policy makers to design strategy needed for the prevention and control of occupational injuries. Sex of workers, safety and health supervision, hours worked per week, cigarette smoking and availability of functional danger signs were significantly associated factors with occupational injury.

\section{Acknowledgements}

We would like to express our heartfelt thank to School of Public Health, Addis Ababa University for financing the project, Ministry of Labour and Social Affairs of Ethiopia, Bureau of Labour and Social Affairs of Addis Ababa City Administration, Managers and head of each working department of selected large scale metal manufacturing industry. Data collectors and study participants are also thanked for their willingness to participate in the study.

\section{Authors' Contributions}

Yitagesu Habtu, Abera Kumie and Worku Tefera had participated from start of ideas to finalization of this manuscript.

\section{Competing Interests}

The authors declare that they have no competing interests.

\section{References}

[1] Nyguyen, T. and Luongo, M. (2009) Occupational Injuries and Prevention Activities in Vietnam. Asia-Pacific Journal of Public Health, 15, 7-10.

[2] International Labor Organization (2005) Decent Work-Safe Work. Geneva.

[3] Finish Institute of Occupational Health, International Labor Organization (2009) System for Collection and Analysis of Occupational Accidents Data. African Newsletter on Occupational Health and Safety, 19, 4-5.

[4] Rongo, L.M.B. (2005) Are Workers in Small Scale Industries in Dar es Salaam Aware of Occupational Ergonomics Principle? African Newsletter on Occupational Health and Safety, 15, 14-16.

[5] Rantnen, J. (2008) Basic Occupational Health Services. African Newsletter on Occupational Health and Safety, 18, 29.

[6] Kortum, E. (2009) Raising Awareness of Stress at Work in Developing Countries. Asia-Pacific News Letter on Occupational Health and Safety, 15, 20-23.

[7] Finish Institute of Occupational Health, International Labor Organization (2005) Women and Psychosocial Hazards They Face at Workplace. African Newsletter on Occupational Health and Safety, 18, 52-53.

[8] Ministry of Labor and Social Affairs (2008) Workrelated Injuries Report from Federal, Amhara and SNNRGs. Department of Occupational Health and Safety at Work, Addis Ababa.

[9] Ministry of Health (2008) Report on Non Communicable Diseases. Department of Disease Prevention and Control, Addis Ababa.

[10] Tadesse, T. and Kumie, A. (2007) Prevalence and Factors Affecting Work Related Injuries among Small and Medium Scale Industries in Gonder Woreda, North Gondor Zone, Amhara Regional State, Ethiopia. Ethiopian Journal of Health Development, 21, 25-34. http://dx.doi.org/10.4314/ejhd.v21i1.10028

[11] Osman, Y. and Kumie, A. (2007) Assessment of Occupational Injuries in Tendaho Agricultural Development S.C, Afar Regional State. AAU, Addis Ababa.

[12] Kiwekete, H.M. (2008) Job Safety Analysis: A Practical Tool for Ensuring Safety of the Workplace. African Newsletter on Occupational Health and Safety, 18, 36-37.

[13] Basu, K., Sahu, S. and Paul, G. (2006) Ergonomics Related Factors Evaluation in Medium Manufacturing Industries in Nepal. Asia-Pacific Journal of Public Health, 13, 56-57.

[14] Manmeet, B.K., Richard, D. and Rinchart, C. (2004) Preventing Occupational Injury in Construction Sector. AsianPacific Newsletter on Occupational Health and Safety, 11, 4-7.

[15] Poosanthanasarn, N., Lohachit, C., Fungladda, W., Sriboorapa, S. and Pulkate, C. (2005) An Ergonomics Intervention Program to Prevent Workers' Injuries in a Metal Auto Parts Factory. Southeast Asian Journal of Tropical Medicine and Public Health, 36, 512-522. 
[16] Kazutaka, K. (2008) Roles of Ergonomic Check Points for Safer and Heathier Work. African Newsletter on Occupational Health and Safety, 18, 24-25.

[17] Nyguyen, T., Thihong, T., Chayut, C. and Jhier, S. (2003) Occupational Health and Safety in Small, Medium Sized and Informal Sector Enterprise in Vietnam. Asia-Pacific Journal of Public Health, 10, 7-10.

[18] Seth, A. and Salminen, S. (2006) Making Sense of Industrial Accidents: Role of Job Satisfaction. Journal of Social Sciences, 2, 127-134. http://dx.doi.org/10.3844/jssp.2006.127.134

[19] Akinori, N., Tomoko, I., Masaya, T., Takashi, H., Minoru, H., Naomi, G., et al. (2006) The Prevalence and Correlates of Occupational Injuries in Small Scale Manufacturing Enterprise. Journal of Occupational Health, 48, 366-376. http://dx.doi.org/10.1539/joh.48.366

[20] Bazheroy, J., Roy, G., Sahai, A. and Soudarssanane, M.B. (2005) Magnitude and Risk Factors of Occupational Injuries in Glass Manufacturing Plant. Journal of Occupational Health, 45, 53-59. http://dx.doi.org/10.1539/joh.45.53

[21] O’Neil, D.H. (2003) Ergonomics in Industrially Developing Countries: Does Its Application Differ from That in Industrially Advanced Countries? Applied Ergonomics, 31, 631-640. http://dx.doi.org/10.1016/S0003-6870(00)00033-8

[22] Bereto, S.M., Swerdlows, A.J. and Smith, P.G. (1998) A Nested Case Control Study of Fatal Work Related Injuries among Brazilian Steel Workers. http://www.ncbi.nlm.nih.gov/pmc/articles/PMC1128985/

[23] Dembe, A., Erickson, J.B. and Delbos, R. (2004) Predictors of Work Related Injuries and Illnesses: National Survey Findings. Journal of Occupational and Environmental Hygiene, 1, 542-550. http://dx.doi.org/10.1080/15459620490478376

[24] Saha, A., Kumar, S. and Vasudevan, D.M. (2007) Occupational Injury Surveillance: A Study in a Metal Smelting Industry. http://www.ijoem.com/text.asp?2007/11/3/103/38458

[25] Addis Ababa Bureau of Trade and Industry (2009) List of Trade and Manufacturing Industries in Ethiopia. Addis Ababa.

[26] Degu, G. and Worku, A. (2010) Differentials of Fertility in North and South Gonder Zone, North West Ethiopia: A Comparative Cross Sectional Study. http://www.biomedcentral.com/1471-2458/8/397

[27] Victoria, C.G., Huttly, S.R., Fuchs, S.C. and Olinto, M.T. (1997) The Role of Conceptual Frameworks in Epidemiological Analysis: A Hierarchical Approach. International Journal of Epidemiology, 26, 224-227. http://dx.doi.org/10.1093/ije/26.1.224

[28] International Labour Organization (1998) Metal Processing and Metal Working Industry. In: Stellman, J., Ed., Encyclopaedia of Occupational Health and Safety, 4th Edition, International Labour Organization, Geneva, 16-25.

[29] Central Statistical Authority (2008) Report on Medium and Large Scale Manufacturing Industry Survey. Addis Ababa.

[30] Caslin, C. (2006) An Investigation into the Measurement of Workplace Injury Severity. Wellington. http://ojs.victoria.ac.nz/LEW/article/view/1609

[31] Faris, K. (1998) Survey of Occupational Safety and Sanitary Conditions in Small Scale Enterprise in Jimma South Western Ethiopia. Ethiopian Journal of Health Development, 12, 183-190.

[32] Senbeto, E. (1991) The Incidence of Injuries and Their Determinants in Akaki Textiles Factory. AAU, Addis Ababa.

[33] Ezenwa, O.A. (2001) A Study of Fatal Injuries in Nigerian Factories. Occupational Medicine, 51, 485-489. http://dx.doi.org/10.1093/occmed/51.8.485 


\section{Acronym and Abbreviation}

AOR: Adjusted Odds Ratio

CI: Confidence Interval

COR: Crude Odds Ratio

CSA: Central Statistical Authority

CSSTHF: Metal Cutting, Scraping, Sorting, Trimming, Haling and Feeding

DALY: Disability Adjusted Life Year

EPI Info: Epidemiological Information

ICOH: International Commission for Occupational Health

ILO: International Labor Organization

MOLSA: Ministry of Labor and Social Affairs

OR: Odds Ratio

PPD/E: Personal Protective Device/Equipment

PPS: Probability Proportional to Size

SNNPRG: Southern Nation Nationalities and Peoples Regional Government

SPSS: Statistical Package for Social Sciences 